\title{
Estudio sobre la construcción cognitiva de la matriz de cambio de base en términos de la Teoría APOE
}

\author{
Esteban Mendoza-Sandoval, Universidad Autónoma de Guerrero (México) \\ Flor Monserrat Rodríguez-Vásquez, Universidad Autónoma de Guerrero (México) \\ Solange Roa-Fuentes, Universidad Industrial de Santander (Colombia) \\ Jesús Romero-Valencia, Universidad Autónoma de Guerrero (México)
}

\begin{abstract}
Estudio sobre la construcción cognitiva de la matriz de cambio de base en términos de la Teoría APOE

\section{Resumen}

Este artículo muestra la construcción de la matriz de cambio de base en álgebra lineal. Con fundamento en la teoría APOE como marco teórico y metodológico, se describen las estructuras y mecanismos mentales para obtener una descomposición genética a partir de las concepciones que estudiantes de una licenciatura de matemáticas tienen sobre dicho concepto. Se aplicó una prueba diagnóstica a 28 estudiantes universitarios (18-21 años de edad). Posteriormente, se realizaron entrevistas semiestructuradas. Los resultados muestran al menos dos trayectorias en la construcción de la matriz de cambio de base, las cuales se diferencian en la interiorización de las acciones mentales.
\end{abstract}

Palabras clave. Descomposición genética, abstracción reflexiva, cambio de base, álgebra lineal.

Study on the cognitive construction of the change of basis matrix in terms of APOS Theory

\section{Abstract}

This article shows the construction of the change of basis matrix in linear algebra. Based on the APOS theory as a theoretical and methodological framework, the structures and mental mechanisms are described to obtain a genetic decomposition based on the conceptions that students have about such a concept. A diagnostic test was applied to 28 undergraduate students (18-21 years of age). Semi-structured interviews were also conducted. The results show at least two trajectories in the construction of change of basis matrix concept, which differ in the internalization of mental actions.

Keywords. Genetic decomposition, reflexive abstraction, change of basis, linear algebra.

\section{Introducción}

Los procesos de enseñanza y aprendizaje de álgebra lineal son complejos debido al carácter abstracto de los conceptos matemáticos, entre ellos, matrices, vectores, sistemas de ecuaciones lineales, espacios vectoriales y transformaciones lineales. Otra razón son los obstáculos epistemológicos. Hillel (2000) detectó al menos dos obstáculos. Uno respecto al pensamiento geométrico ligado a la familiaridad con la geometría y coordenadas estándar; por ejemplo, al considerar vectores y transformaciones en un contexto geométrico, ya que ello obstaculiza la comprensión del concepto de base y el cambio de base en álgebra lineal. Y el otro asociado a un pensamiento algebraico; por ejemplo, al resolver problemas relacionados con la idea de sistemas de ecuaciones lineales en espacios euclidianos, se genera en los estudiantes un obstáculo sobre la aceptación de otros objetos como vectores (funciones, matrices o polinomios). Montiel et al. (2012) corroboran la idea de Hillel (2000) sobre la existencia de un obstáculo de naturaleza conceptual y no solo una dificultad procedimental. 
Hillel y Sierpinska (1994) reportan que estudiantes de pregrado en álgebra lineal tienen una dificultad con la representación de los operadores lineales por matrices en diferentes bases, ya que consideran las columnas de la representación matricial del operador lineal como las imágenes mismas, no considerando la base. Adicionalmente, Selby (2016) menciona que en álgebra lineal se enseña a transitar de un sistema de coordenadas a otro de manera abstracta mediante la matriz de cambio de base.

Desde el enfoque de la teoría APOE, Trigueros et al. (2015) proponen una descomposición genética del teorema de la matriz asociada a una transformación lineal, de donde se infiere que la matriz de cambio de base es un caso particular de este teorema cuando se considera la misma base tanto en el dominio como en el contradominio, ya que se genera la transformación lineal como la identidad sobre el mismo espacio vectorial. Otra investigación es la de Parraguez et al. (2016) quienes proponen una descomposición genética para construir el teorema de cambio de base de vectores: de este teorema se observa que la matriz de cambio de base relaciona las coordenadas de un vector en dos bases diferentes, además, reportan que la construcción de la matriz de coordenadas como proceso no garantiza en los aprendizajes la construcción de coordenadas de un vector como objeto y que la no construcción del concepto coordenadas de un vector como proceso imposibilita la construcción de la matriz de coordenadas, por lo que, en nuestra investigación, consideramos que el vector de coordenadas debe requerirse como objeto y no como proceso. De ahí, la matriz de cambio de base es un concepto necesario para la construcción de otros conceptos.

En este artículo se propone una descomposición genética sobre el concepto matriz de cambio de base en álgebra lineal, por lo que se describen las estructuras y mecanismos mentales asociados a dicho concepto.

\section{Teoría APOE}

La teoría APOE considera que el mecanismo principal en la construcción del conocimiento es la abstracción reflexiva (Arnon et al., 2014; Dubinsky, 1991). Esta teoría se usa como modelo cognitivo para describir cómo se pueden aprender los conceptos matemáticos y para entender cómo se construye el conocimiento matemático. Mediante la descomposición genética se define un modelo hipotético que describe estructuras y mecanismos mentales que un estudiante necesita para aprender un concepto matemático (Arnon et al., 2014). Se deben reconocer relaciones y procesos entre conceptos para construir nuevo conocimiento matemático (Dubinsky, 1991; Trigueros, 2005; RoaFuentes y Oktaç, 2012). Las habilidades para organizar y (re)construir conocimiento matemático se realizan a través de estructuras mentales como acción, proceso, objeto y esquema.

Mientras las estructuras mentales subsisten en un individuo para construir significados en una demanda cognitiva, los mecanismos mentales (interiorización, coordinación, encapsulación, reversión y tematización) tienen como función principal motivar la construcción de las estructuras y permitir transitar entre una y otra o revertir la estructura en un individuo, cuando está frente a una situación matemática.

De acuerdo a Trigueros y Oktaç (2019), las acciones se definen como transformaciones aplicadas a un objeto, es decir, el objeto se manipula o se usa para realizar un cálculo de acuerdo a un algoritmo o procedimiento memorizado, mencionan que los alumnos los perciben como externos, puesto que ellos no pueden justificar los pasos que siguen o predecir el resultado de su aplicación; los procesos se logran cuando se reflexiona sobre una acción o una serie de acciones y estas se repiten de tal forma que puedan 
ser interiorizadas, lo que significa que el alumno comienza a percibir las acciones como internas y pueden omitir pasos y anticipar el resultado de su aplicación; los objetos se alcanzan cuando se tiene la necesidad de transformar un proceso, pues puede llevar al alumno a realizar o imaginar acciones en este y, por lo tanto, encapsularlo justo como un objeto; un esquema es una construcción coherente compuesta de acciones, procesos, objetos y otros esquemas construidos previamente que están relacionados con el mismo concepto matemático o tema.

\section{Metodología}

Se consideraron las componentes del ciclo de investigación: análisis teórico, colección y análisis de datos (Arnon et al., 2014). Para el análisis teórico, se realizó una revisión de libros de texto de álgebra lineal, se identificó cómo se propone la matriz de cambio de base para su enseñanza; adicionalmente, para conocer cómo podría ser construido el concepto por los estudiantes, se entrevistó a dos expertos en la enseñanza del álgebra lineal, profesores con más de 10 años de experiencia impartiendo la asignatura en una licenciatura en matemáticas. Se les preguntó qué conceptos son primordiales para el aprendizaje de la matriz de cambio de base; ambos coincidieron en los conceptos de base ordenada, combinación lineal, conjunto solución de sistema de ecuaciones lineales y vector de coordenadas. Los datos se recolectaron a partir de una prueba diagnóstica y una entrevista semiestructurada.

\subsection{Análisis teórico de la matriz de cambio de base}

La revisión de Álgebra lineal (Hoffman y Kunze, 1973), Introducción al álgebra lineal (Anton, 1994), Fundamentos de álgebra lineal y aplicaciones (Florey, 1980), Álgebra lineal (Grossman, 2008) y Álgebra lineal: Una introducción moderna (Poole, 2011) permitió detectar cómo se presenta el concepto matriz de cambio de base, incluidas definición, nombres y un teorema asociado a su existencia y unicidad (Figura 1).

Las estructuras previas requeridas para la construcción de la matriz de cambio de base son: i) base ordenada como objeto, lo que implica la posibilidad de trabajar con dos bases para un mismo espacio vectorial y actuar sobre ellas mediante la matriz de cambio de base; ii) vector de coordenadas como objeto, implica concebir a las coordenadas del vector $v \in V$ respecto a una base como un vector del espacio vectorial $K^{n}$ sobre el cual puede aplicar acciones específicas (operaciones binarias o considerarlo como una columna de la matriz de cambio de base). Asimismo, del análisis teórico, se infiere la descomposición genética preliminar como sigue: los estudiantes pueden trabajar con bases distintas o iguales sobre el mismo espacio vectorial dado; también pueden calcular la matriz de cambio de base en espacios euclidianos a partir de acciones específicas en las bases (establecen o identifican un orden en las bases y determinan cada vector de la base de salida [inicial] como combinación lineal de la base de llegada y, posteriormente, los estudiantes se quedan con la información de los coeficientes de la combinación lineal y los consideran como las coordenadas de un vector, esto lo hacen para cada vector de la base de salida). Una vez que interiorizan dichas acciones, los estudiantes reflexionan sobre la matriz de cambio de base y tienen un medio para distinguir, en función de las columnas, la diferencia entre las matrices cambio de base $M_{B \rightarrow B_{1}}$ y $M_{B_{1} \rightarrow B}$, además, son capaces de determinar una matriz de cambio de base en cualquier espacio vectorial de dimensión finita. Finalmente, el proceso de matriz cambio de base se encapsula en un objeto, cuando los estudiantes consideran la matriz cambio de base como un elemento del conjunto de matrices $n \times n$ invertibles, donde $n$ depende de la dimensión del espacio vectorial. Esto se presenta cuando los estudiantes deben enfrentar situaciones donde 
necesiten aplicar acciones sobre este nuevo objeto, por ejemplo, usar la matriz de cambio de base para determinar las coordenadas de un vector respecto a otra base.

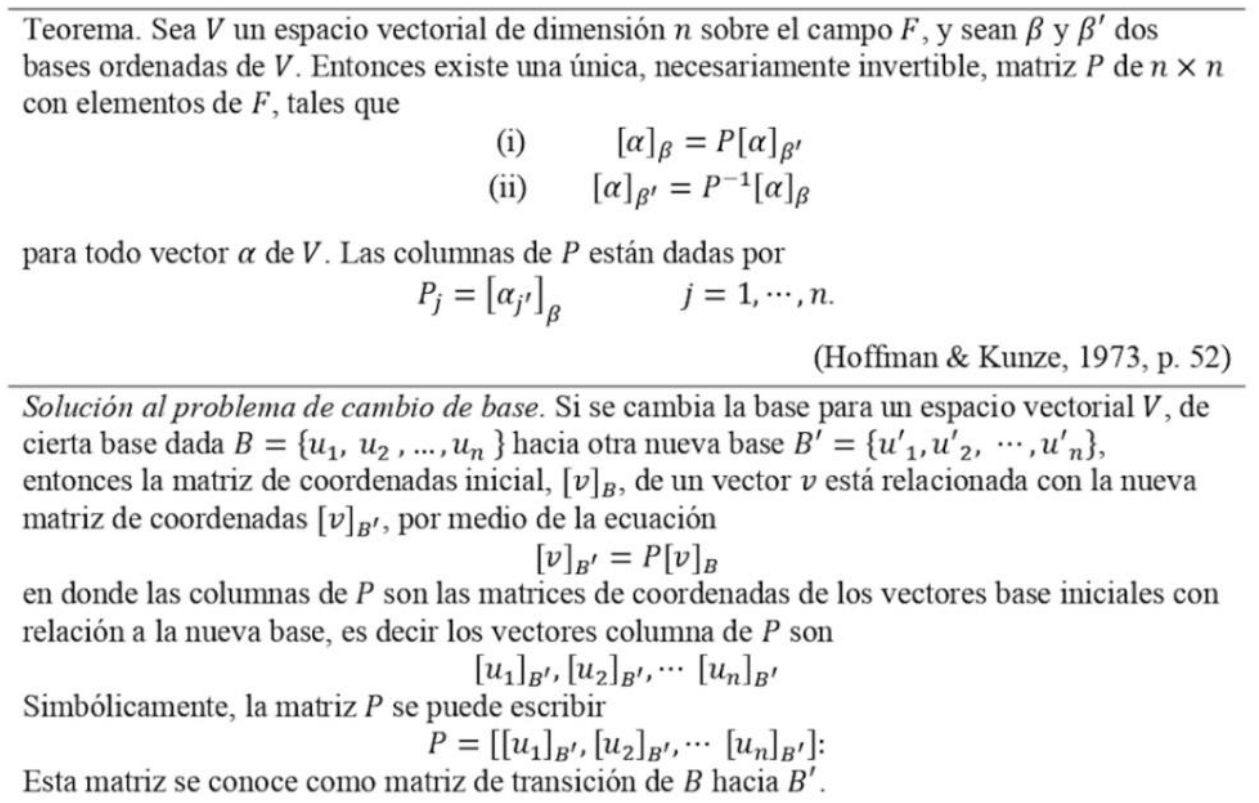

(Anton, 1994, p. 229)

Sean $C_{1}=\left\{X_{1}, X_{2}, \cdots, X_{n}\right\}$ y $C_{1}=\left\{Y_{1}, Y_{2}, \cdots, Y_{n}\right\}$ bases ordenadas para el espacio vectorial $V$, $\mathrm{y}$ sea $I: V \rightarrow V$ la trasformación identidad. La matriz $Q=[I]_{C_{1} C_{2}}=\left(\left[X_{1}\right]_{C_{2}},\left[X_{2}\right]_{C_{2}}, \cdots,\left[X_{n}\right]_{C_{2}}\right)$ Se llama la matriz de cambio de base de $C_{1}$ a $C_{1}$ (porque $Q[X]_{C_{1}}=[X]_{C_{2}}$ ).

(Florey, 1980, p. 271)

La matriz $A$ de $n \times n$ cuyas columnas están dadas por (8) se llama matriz de transición de la base $B_{1}$ a la base $B_{2}$. Esto es,

$$
\begin{aligned}
& \left(u_{j}\right)_{B_{2}}=\left(\begin{array}{c}
a_{i j} \\
a_{2 j} \\
\vdots \\
a_{n j}
\end{array}\right)
\end{aligned}
$$

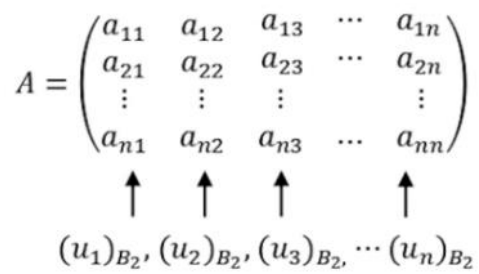

Nota. Si se cambia el orden en el que se escriben los vectores de la base, entonces también debe cambiarse el orden de las columnas en la matriz de transición.

(Grossman, 2008, p. 369)

Sean $B=\left\{u_{1}, u_{2}, \ldots, u_{n}\right\}$ y $C=\left\{v_{1}, v_{2}, \ldots, v_{n}\right\}$ bases para un espacio vectorial $V$. La matriz de $n \times n$ cuyas columnas son los vectores coordenados $\left[u_{1}\right]_{C}, \ldots,\left[u_{n}\right]_{C}$ de los vectores en $B$ con respecto a $C$ se denota $P_{C \leftarrow B}$ y se llama matriz de cambio de base $B$ a $C$. Esto es,

$$
P_{C \leftarrow B}=\left[\left[u_{1}\right]_{C}\left[u_{2}\right]_{C} \ldots\left[u_{n}\right]_{C}\right]
$$

(Poole, 2011, p. 483)

Figura 1. Matriz de cambio de base en textos de álgebra lineal

Posteriormente se diseñó una prueba diagnóstica con los objetivos de: 1) analizar y verificar el tipo de estructuras previas para abordar la construcción del concepto matriz de cambio de base; 2) identificar dificultades asociadas a la comprensión de la matriz de cambio de base; 3) seleccionar a individuos con distintas estructuras mentales sobre el concepto de interés. La prueba se aplicó a 28 estudiantes de la Universidad Industrial de Santander, Colombia, quienes habían tomado por lo menos un curso de álgebra lineal. La aplicación duró 120 minutos. La prueba constó de cinco ejercicios acerca de los 
conceptos coordenadas de un vector y matriz de cambio de base (Figura 2). Fueron problemas típicos en los libros de texto en un curso de álgebra lineal, y fueron validados por dos expertos en la materia como potenciales para promover las construcciones mentales necesarias en la construcción de la matriz de cambio de base.

1. Sea $\beta=\left\{v_{1}, v_{2}, v_{3}\right\}$ una base ordenada de $\mathbb{R}^{3}$ formada por los vectores:

$$
v_{1}=(1,0,-1), v_{2}=(1,1,1), v_{3}=(1,0,0) .
$$

(i) ¿Cuáles son las coordenadas del vector $(5,-5,1)$ en términos de la base ordenada $\beta$ ?

(ii) ¿Cuáles son las coordenadas del vector $(a, b, c)$ en términos de la base ordenada $\beta$ ?

2. Sean las bases ordenadas $\beta=\left\{v_{1}, v_{2}\right\}$ y $\beta^{\prime}=\left\{e_{1}, e_{2}\right\}$ del espacio $\mathbb{R}^{2}$, formadas por los vectores:

$$
v_{1}=(1,2), v_{2}=(2,1) \text { y } e_{1}=(1,0), e_{2}=(0,1)
$$

(i) Encuentre la matriz de cambio de base de $\beta$ a $\beta^{\prime}$.

(ii) Encuentre la matriz de cambio de base de $\beta^{\prime}$ a $\beta$.

3. Dada la matriz de cambio de base $\boldsymbol{M}_{\boldsymbol{\beta}^{\prime} \rightarrow \boldsymbol{\beta}}=\left[\begin{array}{ccc}2 & 2 & 1 \\ 1 & -1 & 2 \\ 1 & 1 & 1\end{array}\right]$ donde las bases ordenadas están definidas como:

$$
\beta=\{(2,0,1),(1,2,0),(1,1,1)\}
$$

$$
\beta^{\prime}=\{(6,3,3),(4,-1,3),(5,5,2)\}
$$

$$
\text { Y sea el vector }\left[\begin{array}{c}
4 \\
-9 \\
5
\end{array}\right]_{\beta^{\prime}}=\left[\begin{array}{c}
1 \\
2 \\
-2
\end{array}\right] \text {. Calcule el vector }\left[\begin{array}{c}
4 \\
-9 \\
5
\end{array}\right]_{\beta} \text {. }
$$

4. Sea la matriz $\boldsymbol{P}=\left[\begin{array}{ccc}1 & 2 & -1 \\ -3 & -5 & 0 \\ 4 & 6 & 1\end{array}\right] \mathrm{y}$, sean los vectores $v_{1}=\left(\begin{array}{c}-2 \\ 2 \\ 3\end{array}\right), v_{2}=\left(\begin{array}{c}-8 \\ 5 \\ 2\end{array}\right) \mathrm{y}$ $v_{3}=\left(\begin{array}{c}-7 \\ 2 \\ 6\end{array}\right)$ vectores de una base ordenada $\beta=\left\{v_{1}, v_{2}, v_{3}\right\}$.

a) Encuentre una base $\beta^{\prime}=\left\{u_{1}, u_{2}, u_{3}\right\}$ para $\mathbb{R}^{3}$ tal que $P_{\beta^{\prime} \rightarrow \beta}$ sea la matriz de cambio de base de $\beta^{\prime}=\left\{u_{1}, u_{2}, u_{3}\right\}$ a $\beta=\left\{v_{1}, v_{2}, v_{3}\right\}$.

5. ¿Qué entiendes por Matriz de cambio de base?

Figura 2. Prueba diagnóstica

Según resultados de la prueba diagnóstica, se seleccionaron seis estudiantes para la entrevista, con evidencia de tener las estructuras previas necesarias para la construcción del concepto matriz de cambio de base para $\mathbb{R}^{2}$. Las entrevistas duraron 130 minutos por estudiante, se grabaron en video y transcribieron. Para triangular datos se analizaron las respuestas de estudiantes a partir de la prueba diagnóstica, en términos de la descomposición preliminar y las concepciones ligadas a la matriz de cambio de base. Se confrontaron estos datos con los de entrevista, confirmando estructuras mentales reconocidas e identificando otras no surgidas en el diagnóstico.

\subsection{La entrevista y su análisis a priori}

La entrevista semiestructurada tuvo por objetivos: 1) validar la descomposición genética preliminar; 2) identificar estructuras y mecanismos que no se evidenciaron en la 
prueba diagnóstica y que son fundamentales en la construcción del concepto matriz de cambio de base. Con fundamento en el análisis teórico, en la entrevista se propusieron seis problemas en diferentes subespacios vectoriales (Figura 3).

En el ejercicio 1, se pide calcular una matriz de cambio de base en el espacio vectorial $\mathbb{R}^{2}$. Se espera que se realicen las acciones siguientes: expresar cada vector de la base de salida como combinación lineal de los vectores de la base de llegada; y con su concepción acción del vector de coordenadas, determinar cada una de las columnas de la matriz solicitada en cada inciso.

1. Sea $V=\mathbb{R}^{2}$ el espacio vectorial definido sobre el campo $\mathbb{R}$ y sean: $B_{1}=\left\{\left(\begin{array}{l}0 \\ 1\end{array}\right),\left(\begin{array}{l}1 \\ 0\end{array}\right)\right\}$, $B_{2}=\left\{\left(\begin{array}{l}1 \\ 1\end{array}\right),\left(\begin{array}{l}2 \\ 1\end{array}\right)\right\}, B_{3}=\left\{\left(\begin{array}{l}1 \\ 0\end{array}\right),\left(\begin{array}{l}0 \\ 1\end{array}\right)\right\}$, bases ordenadas de $\mathbb{R}^{2}$.

a) Encuentre la matriz $\mathbf{P}_{\mathbf{B}_{1} \rightarrow \mathbf{B}_{2}}$.

b) Encuentre la matriz $\mathbf{P}_{\mathbf{B}_{3} \rightarrow \mathbf{B}_{2}}$.

c) Encuentre la matriz $\mathbf{P}_{\mathbf{B}_{\mathbf{2}} \rightarrow \mathbf{B}_{3}}$.

2. Sean las bases $B_{1}=\left\{t_{3}, t_{2}, t_{1}\right\}$ y $B_{2}=\left\{q_{0}, q_{1}, q_{2}\right\}$ bases ordenadas del espacio vectorial $\mathbb{P}_{2}[x]$, definido sobre $\mathbb{R}$. Donde $t_{1}=1, t_{2}=x, t_{3}=x^{2}, q_{0}=1+2 x+x^{2}, q_{1}=2+9 x, q_{2}=3+3 x+4 x^{2}$.

a) Encuentre la matriz cambio de base $B_{1}$ a $B_{2}$.

b) Encuentre el vector de coordenadas de $[p]_{B_{1}}$ con $p=-1+x$ y con ello calcule $[p]_{B_{2}}$.

3. Sean $\mathbf{A}=\left\{a_{1}, a_{2}, a_{3}\right\}$ y $B=\left\{b_{1}, b_{2}, b_{3}\right\}$ bases ordenadas para un espacio vectorial $V$ y suponga que $a_{1}=4 b_{1}-b_{2}, a_{2}=-b_{1}+b_{2}+b_{3}$ y $a_{3}=b_{2}-2 b_{3}$.

a) Encuentre la matriz cambio de base $\mathbf{A}$ a $\mathbf{B}$.

b) Encuentre $[x]_{B}$ para $x=3 a_{1}+4 a_{2}+a_{3}$.

4. Considere la base $B_{1}=\left\{p_{0}, p_{1}, p_{2}\right\}$ ordenada de $\mathbb{R}[x]_{2}$ formada por los polinomios: $p_{0}(x)=$ $1, p_{1}(x)=1+x$ y $p_{2}(x)=(1+x)^{2}$ y sea la matriz: $\mathbf{P}=\left[\begin{array}{ccc}1 & -1 & 0 \\ 0 & 2 & 2 \\ 1 & 0 & -1\end{array}\right]$.

Encuentre los polinomios de la base ordenada $B_{2}=\left\{q_{0}, q_{1}, q_{2}\right\}$, tal que $\mathbf{P}$ sea la matriz cambio de base de $B_{1}$ a $B_{2}$.

5. Considere el espacio vectorial de todos los polinomios de grado menor o igual a $n\left(\mathbb{R}[x]_{n}\right)$ y dos bases ordenadas $B_{1}$ y $B_{2}$ de dicho espacio vectorial. Si se quiere construir la matriz de cambio de base $\mathbf{M}_{\mathbf{B}_{1} \rightarrow \mathbf{B}_{2}}$. ¿Qué tamaño tendrá la matriz? ¿Por qué?

6. ¿Qué entiende por matriz cambio de base? Escriba la definición de este concepto. ¿Dadas dos bases de un espacio vectorial de dimensión finita, siempre es posible definir la matriz cambio de base? ¿Dada una matriz cuadrada cualquiera $\mathbf{A}$, siempre $\mathbf{A}$ representa una matriz cambio de base?

Figura 3. Problemas de la entrevista

En el ejercicio 2, los estudiantes deberán encontrar la matriz de cambio de base $M_{B_{1} \rightarrow B_{2}}$ entre bases ordenadas del espacio $\mathbb{P}_{2}[x]$. Si logran determinarla, tendrán una concepción proceso de la matriz de cambio de base, dado que realizan el trabajo en un espacio vectorial distinto a un espacio euclidiano. Se espera que escriban cada vector de la base $B_{1}$ como combinación lineal de los elementos de la base $B_{2}$ (sin importar el orden en las bases), puesto que además tienen una concepción objeto de base ordenada. Deberán obtener los escalares de la combinación lineal y considerarlos como las coordenadas de un vector, lo cual realizarán para cada vector de la base de salida. Luego deben considerar esas coordenadas como columnas de la matriz. Se busca que los estudiantes apliquen la acción de multiplicar la matriz de cambio de base por el vector de coordenadas, para obtener otro vector de coordenadas escrito en otra base. 
El ejercicio 3 da dos bases $A$ y $B$, conformadas de vectores generales a fin de evitar que los estudiantes realicen sólo cálculos sobre vectores específicos y reflexionen sobre las columnas de una matriz de cambio de base. Con una concepción proceso de base ordenada, se entenderá que cada vector de la base $A$ es combinación lineal de los vectores de la base $B$, y con una concepción objeto del vector de coordenadas se podría establecer que las coordenadas del vector forman una columna de la matriz de cambio de base. Este problema lo hemos relacionado con una concepción proceso de la matriz de cambio de base. Se espera la interiorización de la acción específica siguiente: al estar escrito cada vector de una base como combinación lineal de la otra, se prevé que se interiorice que los vectores de coordenadas son las columnas de la matriz de cambio de base pedida, en este sentido, se forma una concepción proceso de base ordenada. Se busca evidenciar la aplicación de una acción sobre la matriz de cambio de base.

En el ejercicio 4, se necesita una concepción proceso de la matriz de cambio de base y luego regresar sobre una concepción acción de esta misma para considerar la matriz $P^{-1}$ en función de sus columnas. Se debe aplicar una acción específica a la matriz $P$ y calcular $P^{-1}$, la matriz de cambio de base de $B_{2}$ a $B_{1}$. Con una concepción proceso de la matriz de cambio de base podrían deducir lo que representan las columnas de la matriz $P^{-1}$. Con su concepción objeto de base ordenada, podrían deducir los vectores buscados.

El ejercicio 5 relaciona la dimensión del espacio vectorial dado con el orden de la matriz de cambio de base (tamaño) a obtener. Estas son características del concepto de estudio y las asociamos con una estructura objeto. Los estudiantes con una concepción proceso de base ordenada podrían dar bases ordenadas conocidas. Luego, para cada vector definido en la base, este se tiene que escribir como combinación lineal de los elementos de la otra base. Dado que el procedimiento se realiza para $n+1$ vectores de la base de salida, se encuentran $n+1$ coeficientes en la combinación lineal, que resultan ser las coordenadas del vector de coordenadas (interiorización de acciones específicas). Con una concepción objeto del vector de coordenadas los estudiantes podrían tener claro que dicho vector es una columna de la matriz de cambio de base. El orden de la matriz es $n+1 \times n+1$, siendo $n+1$ la dimensión del espacio $\mathbb{P}_{n}[x]$.

El ejercicio 6 busca la definición matemática del concepto de estudio. Las preguntas se plantean con la intención de establecer una reflexión sobre las condiciones esenciales para la existencia de la matriz de cambio de base.

\section{Resultados}

Esta sección se organiza según las estructuras acción, proceso y objeto. Los códigos fueron S15 para estudiante 15, I para entrevistador y Q1a para Pregunta 1 inciso a. Para construir la matriz de cambio de base, se propuso la aplicación de acciones específicas sobre las estructuras objeto de base ordenada y objeto del vector de coordenadas.

\subsection{Sobre la concepción acción de la matriz de cambio de base}

Con una concepción acción, puede haber confusión entre matrices de cambio de base $M_{B \rightarrow B^{\prime}}$ y $M_{B^{\prime} \rightarrow B}$, es decir, se podrían calcular matrices de cambio de base a través de acciones específicas sin la certeza de calcular la matriz requerida. En las respuestas de S13 y S22 ante la Pregunta 2 de la prueba, se ve este tipo de confusión (Tabla 1). 
Tabla 1. Respuestas de S13 y S22 a Pregunta 2 de prueba diagnóstica

Pregunta 2 Sean las bases ordenadas $B=\left\{v_{1}, v_{2}\right\}$ y $B^{\prime}=\left\{e_{1}, e_{2}\right\}$ del espacio vectorial $\mathbb{R}^{2}$, formadas por los vectores: $v_{1}=(1,2), v_{2}=(2,1)$ y $e_{1}=(1,0), e_{2}=(0,1)$. (i) Encuentre la matriz de cambio de base de $B$ a $B^{\prime}$. (ii) Encuentre la matriz de cambio de base de $B^{\prime}$ a $B$.

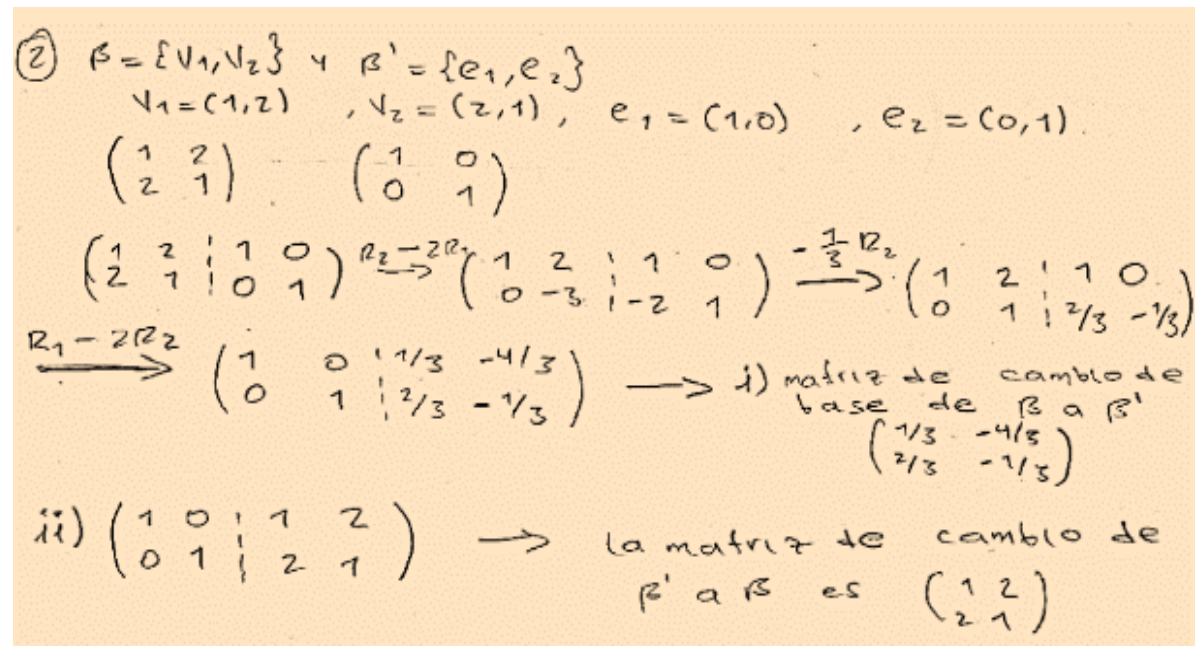

Figura 4. Procedimiento de S13 para calcular una matriz de cambio de base

$$
\begin{aligned}
& \text { (2) } \beta=\left\{\begin{array}{ll}
1 & 2 \\
2 & 1
\end{array}\right\} \quad \beta^{\prime}=\left\{\begin{array}{ll}
1 & 0 \\
0 & 1
\end{array}\right\}
\end{aligned}
$$

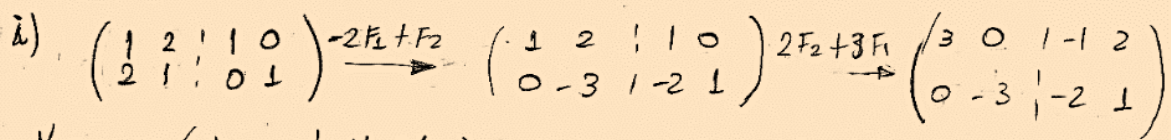

$$
\begin{aligned}
& \underset{-1 / 3 F_{2}}{\stackrel{1 / 3 F_{1}}{\longrightarrow}}\left(\begin{array}{cc|cc}
1 & 0 & -1 / 3 & 2 / 3 \\
0 & 1 & 2 / 3 & -1 / 3
\end{array}\right) \quad M_{\beta \rightarrow \beta^{\prime}}=\left(\begin{array}{cc}
-1 / 3 & 2 / 3 \\
2 / 3 & -1 / 3
\end{array}\right)
\end{aligned}
$$

Figura 5. Procedimiento de S22 para calcular una matriz de cambio de base

S13 y S22 construyeron dos matrices al considerar los vectores de $B$ y $B^{\prime}$ como vectores columnas: $\left(\begin{array}{ll}1 & 2 \\ 2 & 1\end{array}\right),\left(\begin{array}{ll}1 & 0 \\ 0 & 1\end{array}\right)$. Su procedimiento sugirió hacer una matriz aumentada donde colocan en la parte izquierda y derecha las matrices anteriores. Conocían que, al reducir la parte izquierda a la identidad, en la parte derecha tendrían una matriz de cambio de base. Esta forma de proceder como algoritmo está bien al hallar una matriz de cambio de base, pero no se interioriza que las columnas de la matriz que obtienen son las coordenadas de los vectores de $B^{\prime}$ escrito como combinación lineal de los vectores de $B$. Confunden en ambos incisos la matriz requerida. S15 procedió igual, pero de manera correcta y siendo explícitas las acciones sobre las bases, es decir, considerar los vectores transpuestos de las bases y ponerlos como columnas para obtener una matriz para ambas bases, además de construir una matriz ampliada y aplicar operaciones elementales para reducirla y obtener una matriz de cambio de base (Tabla 2).

Otra acción específica sobre las bases ordenadas fue expresar los vectores de la base inicial como combinación lineal de la base nueva. S28 calculó así la matriz de cambio de base. Dijo realizar la acción específica equivocadamente y corregirla (Tabla 3). 
Tabla 2. Fragmento de entrevista de S15

\section{Diálogo}

S15: Entonces quiero la matriz de cambio de $B_{1}$ a $B_{2}$, entonces la matriz que voy a colocar aquí es $B_{2} y$ aquí $B_{1}$. Ahora lo que tengo que hacer es llevar está matriz a la forma escalonada reducida. ¿Sí? [señaló 1, Fig. 6] Y lo que me queda aquí es la matriz de transición de $B_{1}$ a $B_{2}$ [señaló 2, Fig. 6].

I: Entonces vas llevar esto a la identidad [señaló 1, Fig. 6] ¿Y lo que te queda de este lado que va a ser? [señaló 3, Fig. 6].

S15: La matriz de transición de $B_{1}$ a $B_{2}$.

\section{Representaciones}

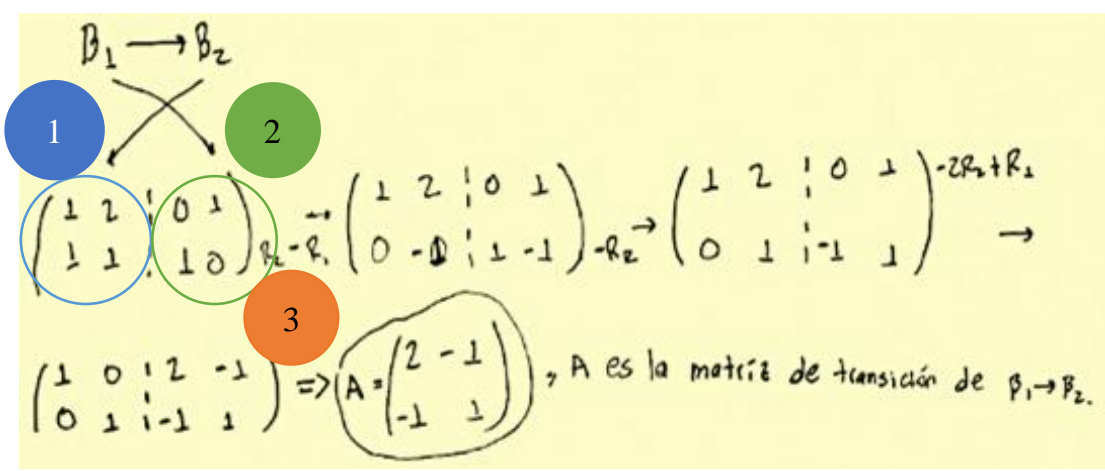

Figura 6. Procedimiento de S15 para calcular una matriz de cambio de base

Tabla 3. Fragmento de entrevista de $S 28$

Diálogo

I: Ok, ¿Y por qué así? ¿Por qué no tomar los vectores de la base $B_{2}$ y expresarlos como combinación lineal de la base $B_{1}$ ?

S28: Porque... me están pidiendo es utilizar estos vectores [señaló $B_{1}$ ], para transformar, para utilizar esta base, para hallar la matriz tal que me sirva para cambiar de acá a acá [señaló vectores de $B_{1}$ a $B_{2}$ ]. Entonces necesito transformar esos vectores en términos de esta base, o sea [señaló vectores de $B_{2} a B_{1}$ ].

I: Sí, es cierto, tienes razón en el sentido de que una matriz de cambio de base toma los vectores de una base y los expresas como combinación lineal de la otra base. Ahora el problema aquí es cuáles. ¿Cuáles vas a tomar estos o estos? [S28 señaló vectores de $B_{1}$ y $B_{2}$ ].

S28: Escribir una base como combinación lineal de la otra. La verdad sí, pues sí, eso es lo que me genera duda de la base $B_{1}$ a la base $B_{2}$ siempre lo he hecho de una forma muy mecánica y no me había puesto a pensar en eso. Luego algunos autores manejan esta notación y le ponen la flechita al revés. ¡A h pues sí! porque yo necesito hacer la transición de base $B_{1}$ a base $B_{2}$.

I: ¿Entonces?

S28: Entonces lo que tendría que hacer es expresar los vectores de la base $B_{1}$ en términos de la base $B_{2}$.

I: Ok, a ver. ¿Qué dijiste? Necesitas expresar estos [S28 señaló $\left.B_{1}\right]$.

S28: En términos de esta base [S28 señaló $B_{2}$ ], y fue todo lo contrario a lo que hice (Figura 7). Voy a escribir la correcta (Figura 8). 


\section{Representaciones}

$$
\begin{aligned}
\left(\begin{array}{l}
1 \\
1
\end{array}\right)=\alpha\left(\begin{array}{l}
0 \\
1
\end{array}\right)+\beta\left(\begin{array}{l}
1 \\
0
\end{array}\right) \quad \beta=1, \alpha=1 \\
\left(\begin{array}{l}
2 \\
1
\end{array}\right)=\alpha\left(\begin{array}{l}
0 \\
1
\end{array}\right)+\beta\left(\begin{array}{l}
1 \\
0
\end{array}\right) \quad \beta=2, \alpha=1 \\
\rho\left(1^{2}\right) \quad p=\left(\begin{array}{l}
1 \\
1
\end{array}\right)
\end{aligned}
$$

Figura 7. Primera respuesta de S28 a Q1a

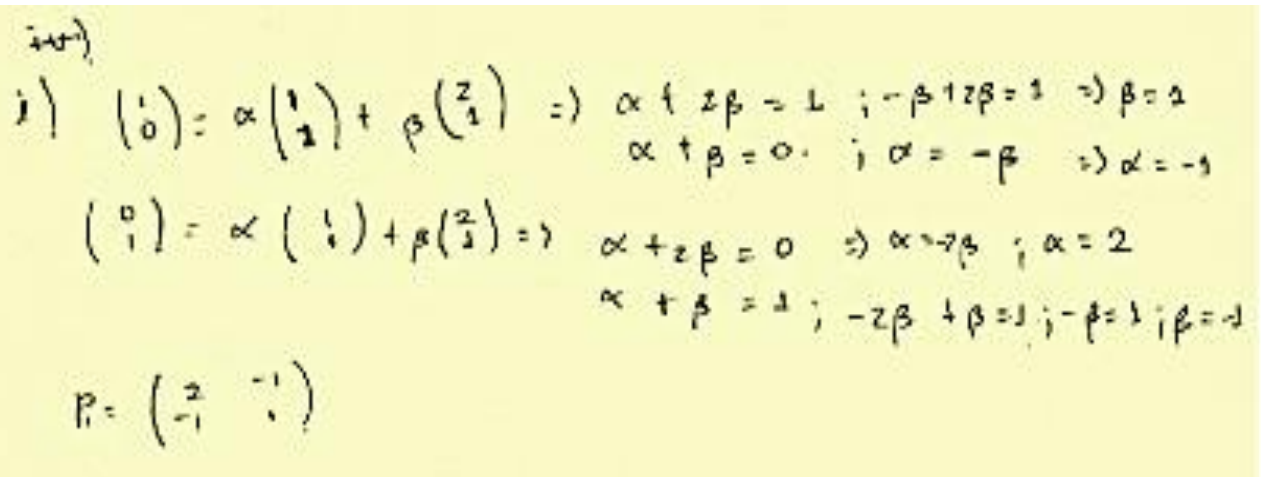

Figura 8. Respuesta final de S28 a Q1a

\subsection{Sobre la concepción proceso de la matriz de cambio de base}

Se alcanzó la interiorización en algunos estudiantes al identificar que las columnas de la matriz de cambio de base estaban relacionadas con la escritura de los vectores de la base inicial como combinación lineal de los vectores de la base final. Algunos estudiantes reconocieron el tamaño de la matriz sin resolver el problema y previeron que el tamaño de la matriz depende del número de elementos de la base y la relación del producto de matrices, por lo que reconocieron el efecto del orden en las bases (Tabla 4). S20 determinó el tamaño de una matriz de cambio de base sin calcular la matriz a partir de la interiorización de acciones específicas y la multiplicación de matrices.

Tabla 4. Fragmento de entrevista de $S 20$

\section{Diálogo}

S20: Listo, sin tener que escribir esto [señaló vectores de base $B_{1}$ ]. Monto los que son, además me están diciendo que son bases, esta genera [señaló base $B_{1}$ ] todos los polinomios en $x$ de segundo grado y son independientes... esta también genera a todos los polinomios de segundo grado en $x$ y también son linealmente independientes [señaló base $B_{2}$ ]. Son tres componentes entonces... esto es de tamaño... tres por uno y este es tres por uno también... por lo tanto... este es tres obligatoriamente. [:] S20: Bueno ya no es dos por dos como el anterior, ahora es tres por tres (Figura 9). 


\section{Representaciones}

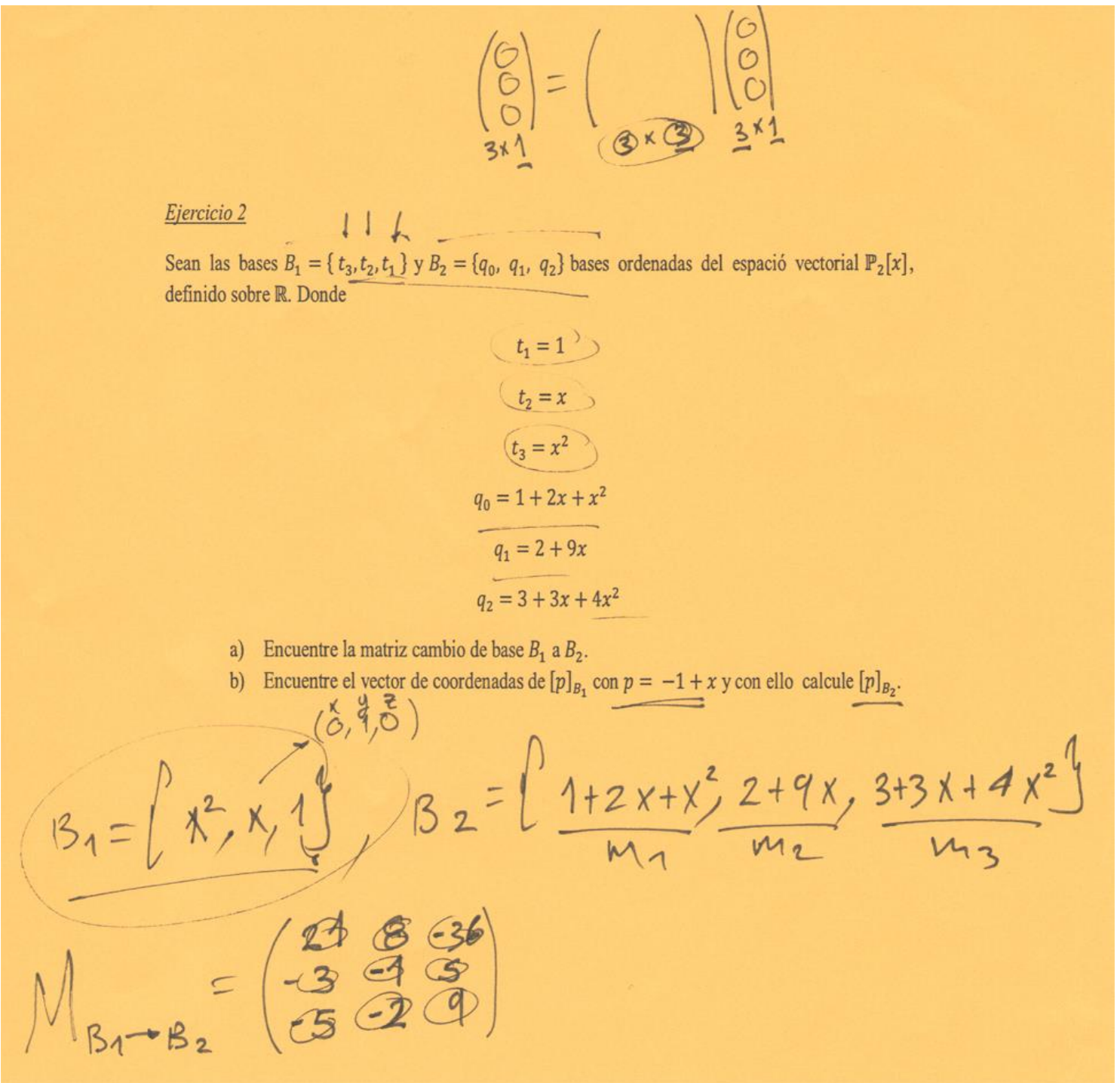

Figura 9. S20 determina el tamaño de la matriz de cambio de base

Cuando se interiorizan las acciones, se puede alcanzar una estructura proceso, que se caracteriza por todas las propiedades que permiten reflexionar y reconocer las características de la matriz de cambio de base, por ejemplo, interiorizar qué representa cada columna; la diferencia entre $M_{B_{1} \rightarrow B_{2}}$ y $M_{B_{2} \rightarrow B_{1}}$; que el orden de las columnas de la matriz de cambio de base depende del orden de los vectores de la base inicial; que el conjunto de dichas columnas son linealmente independientes; más aún, que son una base para el espacio vectorial $K^{n x 1}$, siendo $K$ el campo sobre el cual se ha definido el espacio vectorial. S15 primero tuvo la idea de usar un algoritmo para resolver Q3a, utilizó el espacio vectorial de polinomios de grado menor o igual a dos (Tabla 5). 
Tabla 5. Fragmento de entrevista de S15

\section{Diálogo}

I: ¿Qué es lo que te dan?

S15: Me dan dos bases de un espacio vectorial $V$, me dicen que los vectores del espacio vectorial A están en términos, por decirlo así, en términos de $B$, pues sí, porque $a_{1}=4 b_{1}-$ $b_{2}$ pero estos son vectores de esta base [señaló base $B$ ].

I: Ahora, si yo te pido la matriz de cambio de base de A a B. ¿Cuál es? ¿Va a ser aquella matriz que, qué?

S15: Que... tenga como columnas los vectores de coordenadas de A en términos de B.

I: ¿Ajá y entonces? ¿Cómo encuentras eso?

S15: Con un algoritmo o un sistema de ecuaciones.

I: Ok. Pero cuando usas tu algoritmo. ¿Por qué pones que b es $(1,0,0)$ ? [señaló 4, Fig. 10]. S15: Sí, es que... yo asumí, creo que está mal asumir, eso, que el espacio vectorial es un espacio vectorial de polinomios grado dos.

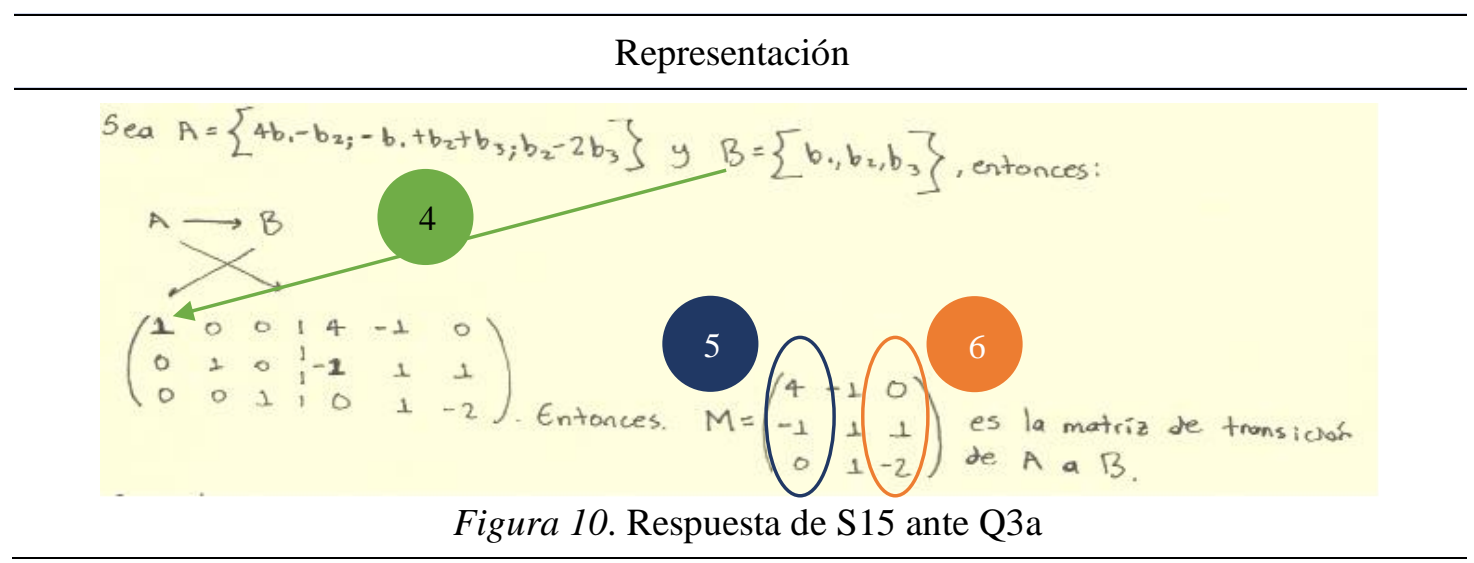

S15 reflexionó respecto a que los vectores de la base inicial se escriben como combinación lineal de los vectores de la base final para obtener los vectores de coordenadas, por lo que alcanzó una interiorización sobre su acción. Así, S15 alcanzó una estructura proceso de la matriz de cambio de base, dado que en su respuesta manifestó que las columnas de una matriz de cambio de base son vectores de coordenadas y la acción específica para obtener cada columna de la matriz. Esto lo logró independientemente del espacio vectorial (Tabla 6).

Tabla 6. Fragmento de entrevista de S15

Diálogo

I: Ok, pero a ver. ¿Qué información tenemos? Tenemos dos bases ordenadas de un espacio vectorial $V$. ¿Qué más te dice?

S15: Que la base A en términos de B.

I: ¿Y eso te ayuda en algo?

S15: Yo creo que sí.

I: ¿En qué? ¿Qué significa que $a_{1}$ lo puedas escribir como $4 b_{1}-b_{2}$ ?

S15: Que el vector $a_{1}$ está en términos de B, o sea, este vector que acabo de escribir. iAy sí, ya miré! Este es el vector $a_{1}$ que pertenece a $A$. ¿Sí?

I: ¿Sí?

S15: $Y$ está en combinación lineal con $b_{1}$ y $b_{2}$ que son vectores de $B_{1}$. Entonces, este vector uno está en combinación lineal de $B$, pues sí es obvio, no, pero si es evidente porque $B$ es base. 
Igual pasa para $a_{2}$ y $a_{3}$ entonces como $a_{1}$ está en términos de $B, a_{2}$ también y $a_{3}$ también, me están queriendo decir que, si yo coloco los vectores de coordenadas como columnas estoy hallando la matriz de transición, es decir, este vector [señaló 5, Fig. 9]. Es el vector de coordenadas de $a_{1}$ en términos de $B, y$ este es el vector de coordenadas de $a_{2}$ en términos de $B$ [señaló 6, Fig. 10]... ¿ ¿Sí... Bueno, entonces lo escribo (Fig. 11).

\section{Representación}

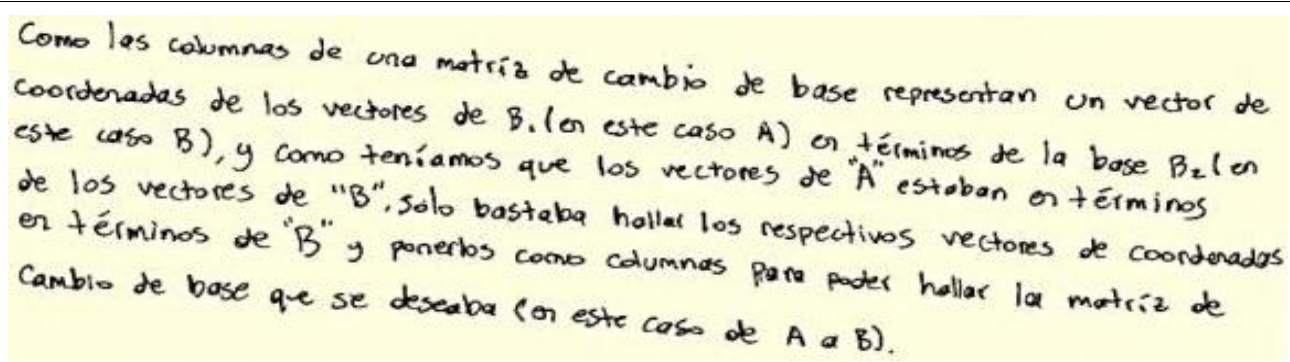

Figura 11. Respuesta de S15 a Q3a

Como indicó el análisis a priori de Q3, con una estructura objeto de base ordenada se podría establecer que cada vector de la base $A$ es combinación lineal de los vectores de la base $B$ y con una concepción objeto del vector de coordenadas se establecería cada columna de la matriz. A diferencia de S15, S9 trabajó sobre un espacio vectorial cualquiera de dimensión finita y fijó la matriz de cambio de base requerida (Tabla 7).

Tabla 7. Fragmento de entrevista de $S 9$

\section{Diálogo}

S9: ... Sería la del primer punto, ya que ya me están dando la combinación lineal de cada elemento ordenado a la otra base [Fig. 12]

I: ¿Eso, lo puedes escribir?

S9: Sí, ya que sé las combinaciones lineales de cada elemento de la base A y la de los elementos de la base B entonces su representación serán los coeficientes en su orden [Fig. 13].

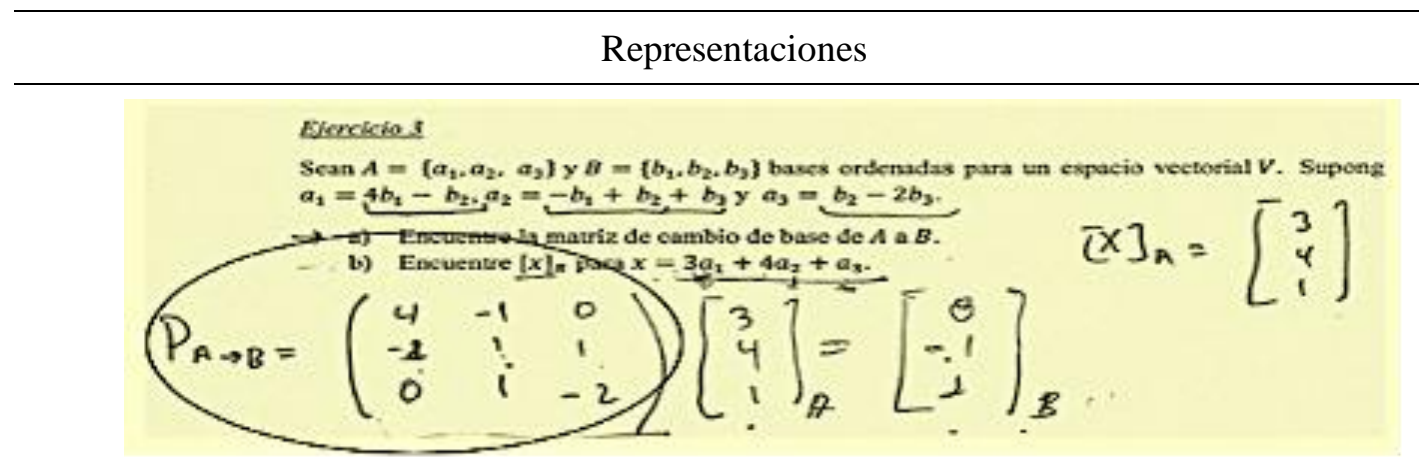

Figura 12. Respuesta de S9 a Q3a

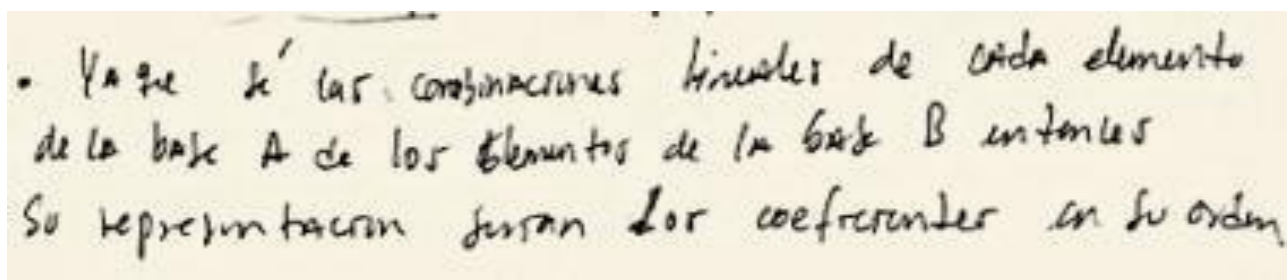

Figura 13. Reflexión de S9 en Q3a 


\subsection{Sobre la concepción objeto de la matriz de cambio de base}

Cuando se tiene la necesidad de dejar estático el proceso de la matriz de cambio de base, se pueden establecer propiedades y características sobre este concepto, pero antes se debe encapsular el proceso en objeto. S15 procedió tal como se describió en el análisis a priori de Q5 y no tuvo dificultad para determinar el tamaño de la matriz de cambio de base, que reconoció como matriz cuadrada (Tabla 8).

Tabla 8. Fragmento de entrevista de S15

\section{Diálogo}

I: ¿Y cuántos elementos tienen las bases?

S15: $n+1$, sí, $n+1$.

I: Ajá. ¿Y eso qué quiere decir?

S15: Que el tamaño de la matriz debe $\operatorname{ser}(n+1) \times(n+1)$.

I: ¿Por qué?

S15: Porque la base tiene $n+1$ vectores.

I: Ok, una base de aquí tiene $(n+1)$ y de ahí, ¿cómo concluyes que el tamaño de la matriz es $(n+1) \times(n+1)$ ?

S15: Entonces al hallar la matriz de transición de $B_{1}$ a $B_{2}$, lo que estoy haciendo es poner este vector en términos de estos vectores y ponerlos como columnas, entonces este primer vector tendrá $n+1$ componentes, y este otro vector tendrá $n+1$ componentes. Entonces al hallar el vector de coordenadas también va a tener $n+1$ componentes, porque son los coeficientes de la combinación lineal. Entonces al poner mis $n+1$ vectores así, va a haber $n+$ 1 vectores como columnas. ¿Sí? Y cada vector tiene $n+1$ componentes, o sea va a haber $n+1$ filas.

I: Ok. ¿Y entonces por eso tendría el tamaño de?

S15: $(n+1) \times(n+1)$ (Fig. 14).

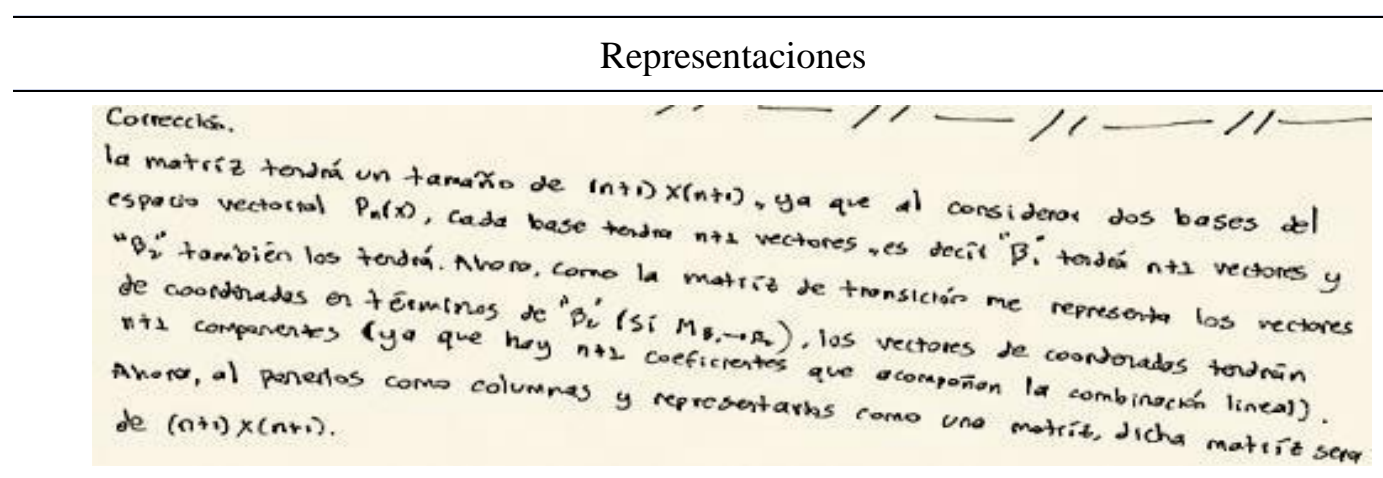

Figura 14. Respuesta de S15 a Q5

S28 y S9 consideraron la matriz de cambio de base como regla de correspondencia (Figura 15). Es decir, consideraron una función $f$ definida como el producto de una matriz de cambio de base por un vector de coordenadas, que recibe como entrada un vector de coordenadas y devuelve un vector de coordenadas respecto a otra base. 


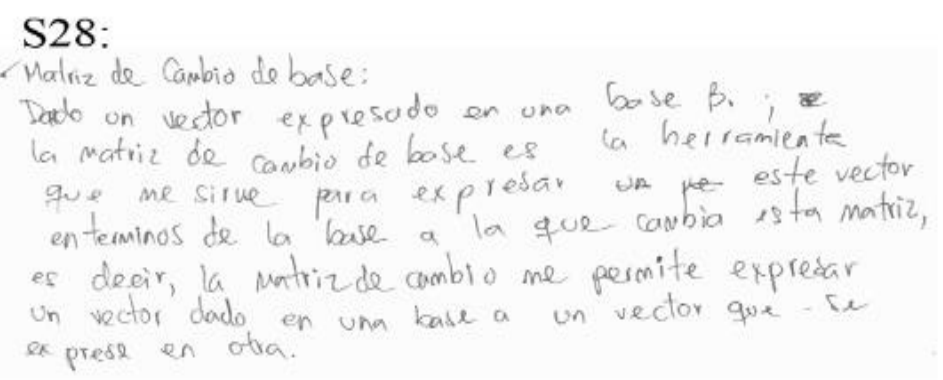

S9:

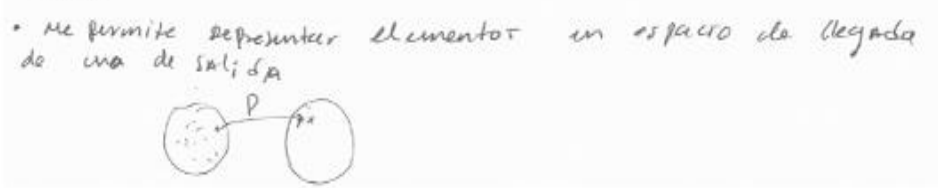

Figura 15. Matriz de cambio de base vista como regla de correspondencia

Una vez que el estudiante encapsula el proceso de la matriz de cambio de base en un objeto, se pueden aplicar acciones específicas; en Q3, S20 consideró la matriz de cambio de base $M_{B_{1} \rightarrow B_{2}}$ como objeto y la utilizó para determinar el vector de coordenadas respecto a la base $B_{2}$ del vector dado (Tabla 9).

Tabla 9. Fragmento de entrevista de S20

\section{Diálogo}

S20: Como yo tengo la matriz puedo aplicar... ¿tengo a $M$ y va de donde a dónde? Tengo las coordenadas en esto... ilisto! [ver 1, Fig. 16]

I: ¿Cómo sabes que este resultado es correcto? [señaló 2, Fig. 16]

S20: Hay una forma de verificar este resultado, pues remplazando esto, o sea... Resolver este... donde está $B_{2}$.... Entonces llamemos a esto $m_{1}, m_{2}, m_{3}$ [escribió 3, Fig. 17] ... Esto tendría que ser igual [...] al vector... a... [escribió 4, Fig. 17]. Estas componentes me van a dar el vector $P$.

\section{Representaciones}

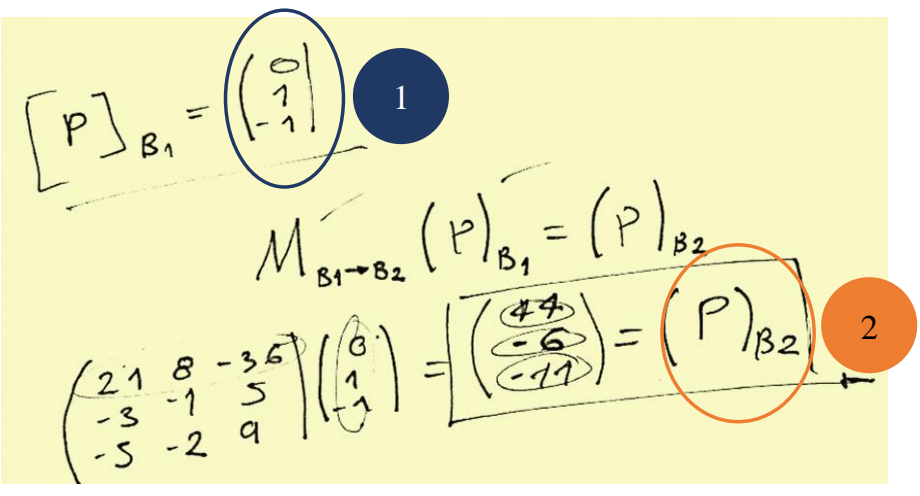

Figura 16. Respuesta de S20 a Q2a parte 1 


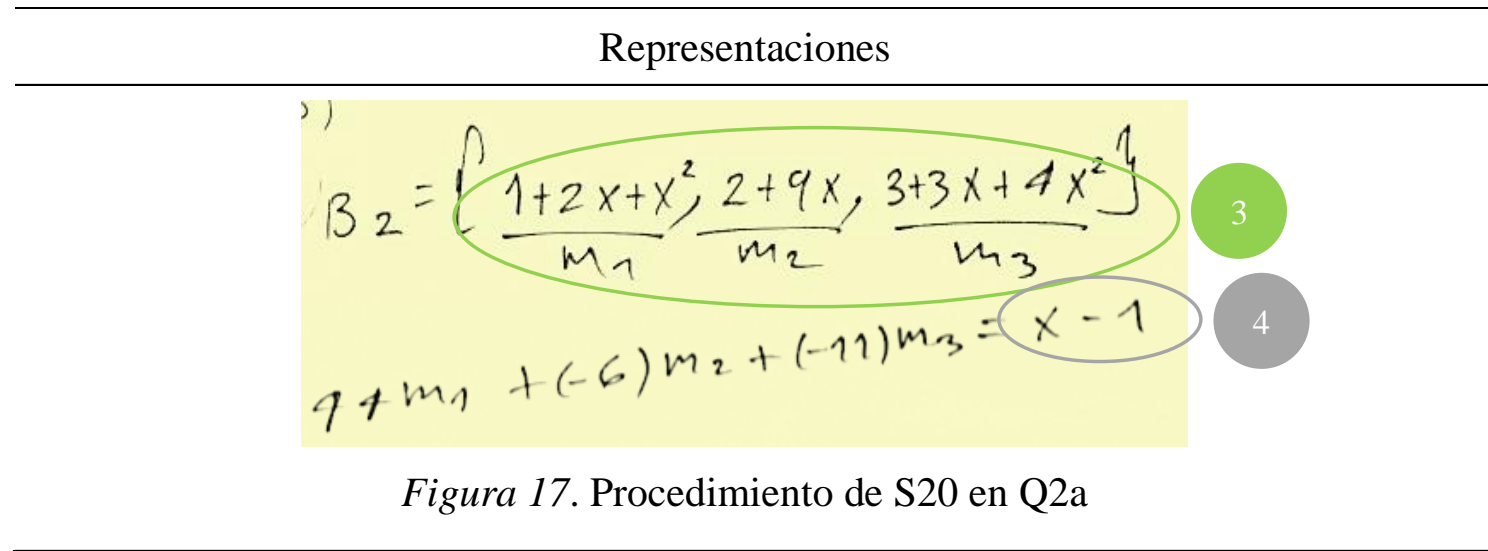

S15 realizó la siguiente acción específica sobre la matriz de cambio de base: multiplicó esta matriz por un vector de coordenadas para obtener un vector de coordenadas en otra base. Luego mostró conocer que la matriz de cambio de base opera con vectores de coordenadas y de que hay relación de correspondencia (Tabla 10).

Tabla 10. Acción específica aplicada a matriz de cambio de base

\section{Diálogo}

S15: Puedo rayar esto para acordarme. Ya me acordé. Listo [escribió 1, Fig. 18]. I: ¿Esa notación $(p)_{B_{1}}$ ? [señaló 1, Fig. 18]

S15: Significa escribir el vector $p$ en términos de la base $B_{1}$. Buscar los coeficientes de la combinación lineal. ¡Aja! Tengo que enunciar el teorema, es que no me acuerdo del nombre del teorema. I: Si quieres, solo enuncia la propiedad que vas a usar para encontrar.

S15: Pues es que hay un teorema que dice que un vector en términos de... [escribió 2, Fig. 18]. ¿Tendría que demostrar eso?

I: ¿Cuál?

S15: Esto [señaló 2, Fig. 18] porque no me acuerdo.

I: La demostración no, pero te voy a preguntar cómo estás seguro de que precisamente te resulta el otro vector $[p]_{B_{2}}$ ¿Cómo me das un argumento donde yo te diga, te creo? ... porque ahí aparece mágicamente ¿no? ¿Y por qué precisamente así y no poner $B_{2}$ aquí y acá el $B_{1}$ ?

S15: Sí, yo lo entiendo, pero espere. Porque la matriz trabaja con coordenadas de vectores y esas tienen que ser las del vector en la base inicial.

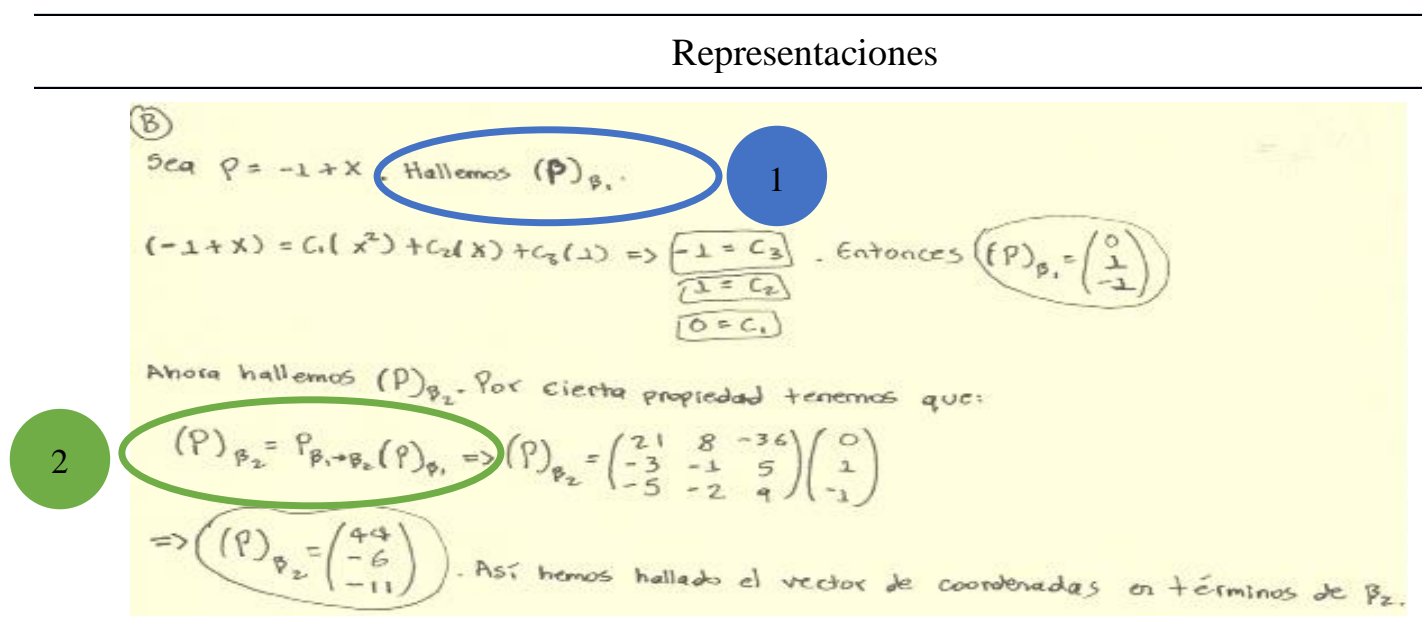

Figura 18. Acción específica sobre matriz de cambio de base 


\section{Conclusiones}

Se identificaron dos trayectorias (Arnon et al., 2014) en la construcción de la matriz de cambio de base, es decir, dos caminos de aprendizaje que siguieron los estudiantes en la construcción del concepto. Estas trayectorias surgieron de la organización de conceptos, estructuras y mecanismos mentales que fueron requiriendo los estudiantes para resolver la prueba diagnóstica, y de reflexionar en la entrevista sobre el problema de escribir los vectores de una base en términos de los vectores de otra base en determinado espacio vectorial. La principal diferencia entre las trayectorias radica en la interiorización de acciones; mientras que en la primera trayectoria los estudiantes deben escribir los vectores de la base inicial como combinación lineal de la base final e interiorizar en un vector de coordenadas, en la segunda trayectoria, al escribir como columnas los vectores de las bases dadas, deben interiorizar qué columnas formadas con los vectores de las bases van del lado izquierdo y derecho de una matriz ampliada. La presentación de ambas trayectorias es una aportación del estudio, puesto que señalan dos caminos de aprendizaje por los que es asequible la comprensión de un concepto en matemáticas. La evidencia de la primera trayectoria en la construcción está en las Tablas 3, 4, 6, 7, 8 y 9, mientras que la evidencia de la segunda trayectoria está en las Tablas 1, 2, 3, 5, 6, 7 y 10. En algunas partes son coincidentes debido a los mecanismos que se requirieron sobre las estructuras. Ambas trayectorias son fundamentales para delinear la descomposición genética de la matriz de cambio de base.

Respecto a las estructuras mentales que se externaron, dos de ellas se habían considerado desde el análisis preliminar, no obstante, se identificó una mas: i) base ordenada como objeto, esta estructura implicó la posibilidad de trabajar con dos bases para un mismo espacio vectorial y actuar sobre estas bases mediante la matriz de cambio de base, además, el estudiante reflexionó sobre el orden de la base $B$, decidir cuál será dicho orden y establecer si el vector dado $v$, o cualquier conjunto de vectores de un espacio vectorial, puede escribirse como una combinación lineal de los elementos de $B$; ii) vector de coordenadas como objeto, implicó concebir a las coordenadas del vector $v \in V$ respecto a una base como un vector del espacio vectorial $K^{n}$, sobre el cual el estudiante logró aplicar acciones específicas (operaciones binarias); iii) matriz como proceso, implicó considerar a la matriz como un arreglo ordenado de filas o columnas. Respecto a los mecanismos mentales, destaca el de interiorización, dado que, mientras por un lado se deben escribir los vectores de la base inicial como combinación lineal de la base final e interiorizar en un vector de coordenadas, por otro, al escribir como columnas los vectores de las bases dadas se debe interiorizar qué columnas formadas con los vectores de las bases van de lado izquierdo y derecho de una matriz ampliada.

A continuación, se describe cada trayectoria y finalmente la descomposición genética del concepto matriz de cambio de base.

Primera trayectoria en la construcción de la matriz de cambio de base. El estudiante desencapsula el objeto de base ordenada en un proceso y con dicha concepción puede escribir los vectores de la base de salida como combinación lineal de los vectores de la base de llegada. Cada proceso de las combinaciones lineales se coordina con el proceso coordenadas de un vector y cada nuevo proceso se encapsula en un vector de coordenadas. Este objeto se desencapsula en el proceso columna de una matriz el cual se coordina con el proceso de matriz en el proceso matriz de cambio de base. Finalmente, el proceso de matriz de cambio de base se encapsula en un objeto cuando se considera la matriz de cambio de base como elemento del conjunto de matrices cuadradas e invertibles y se 
logra considerar a dicha matriz como una función definida como producto de una matriz de cambio de base por un vector de coordenadas (Fig. 19).

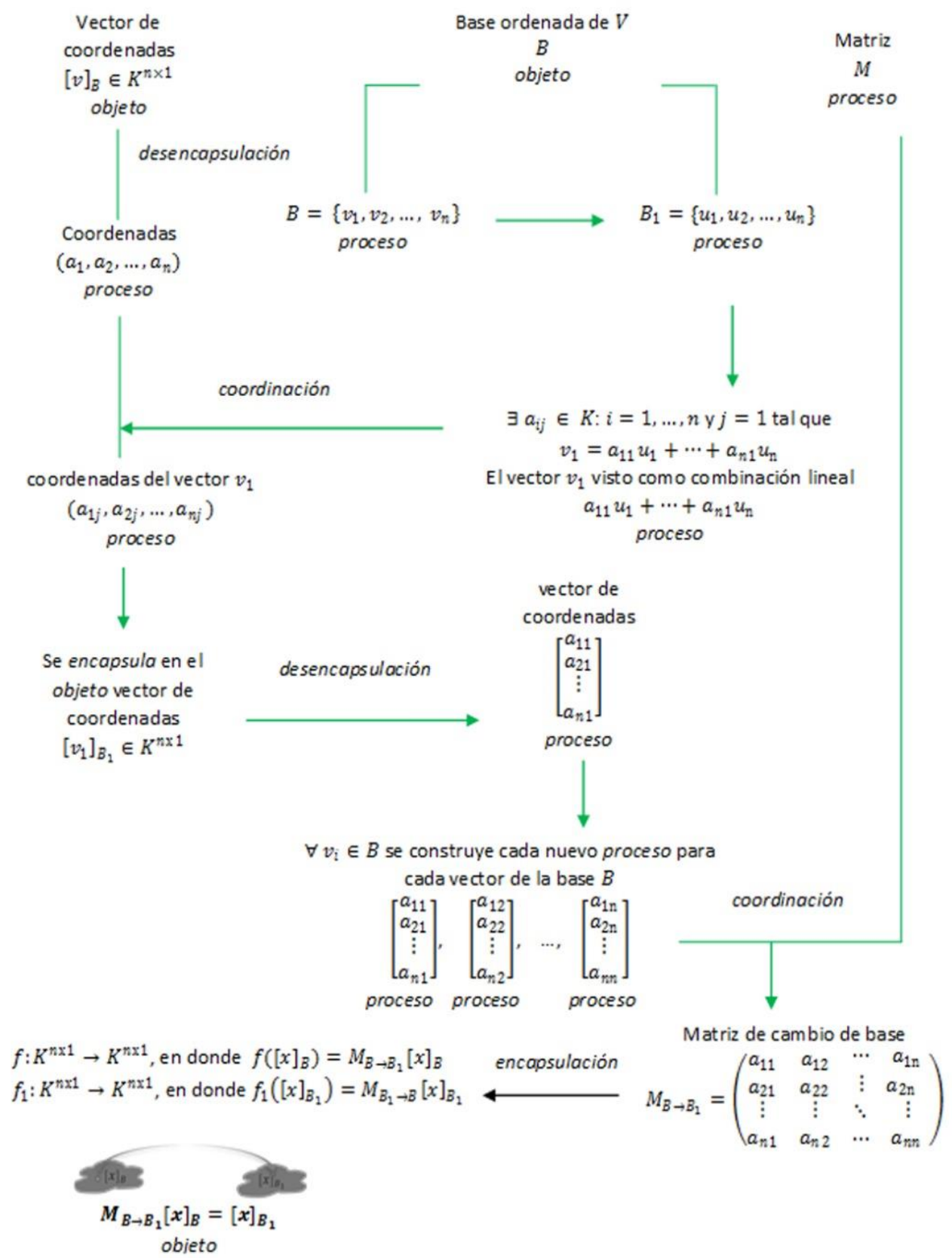

Figura 19. Primera vía de construcción de matriz de cambio de base

Segunda trayectoria en la construcción de la matriz de cambio de base. El estudiante primero debe identificar el orden en la base para decidir cuál vector va primero, cuál va segundo y así sucesivamente. Se aplica la acción específica de escribir las coordenadas de vectores de ambas bases respecto a la base canónica del espacio vectorial dado. Se coordinan los procesos matriz y vector de coordenadas en el proceso de una matriz ampliada (del lado izquierdo se colocan los vectores de coordenadas de base llegada y, del derecho, los vectores de coordenadas de base salida). Se encapsula el proceso en el $o b$ jeto matriz para aplicar el algoritmo de eliminación de Gauss-Jordan, quedando del lado izquierdo la identidad y del derecho una matriz de cambio de base (Fig. 20). 


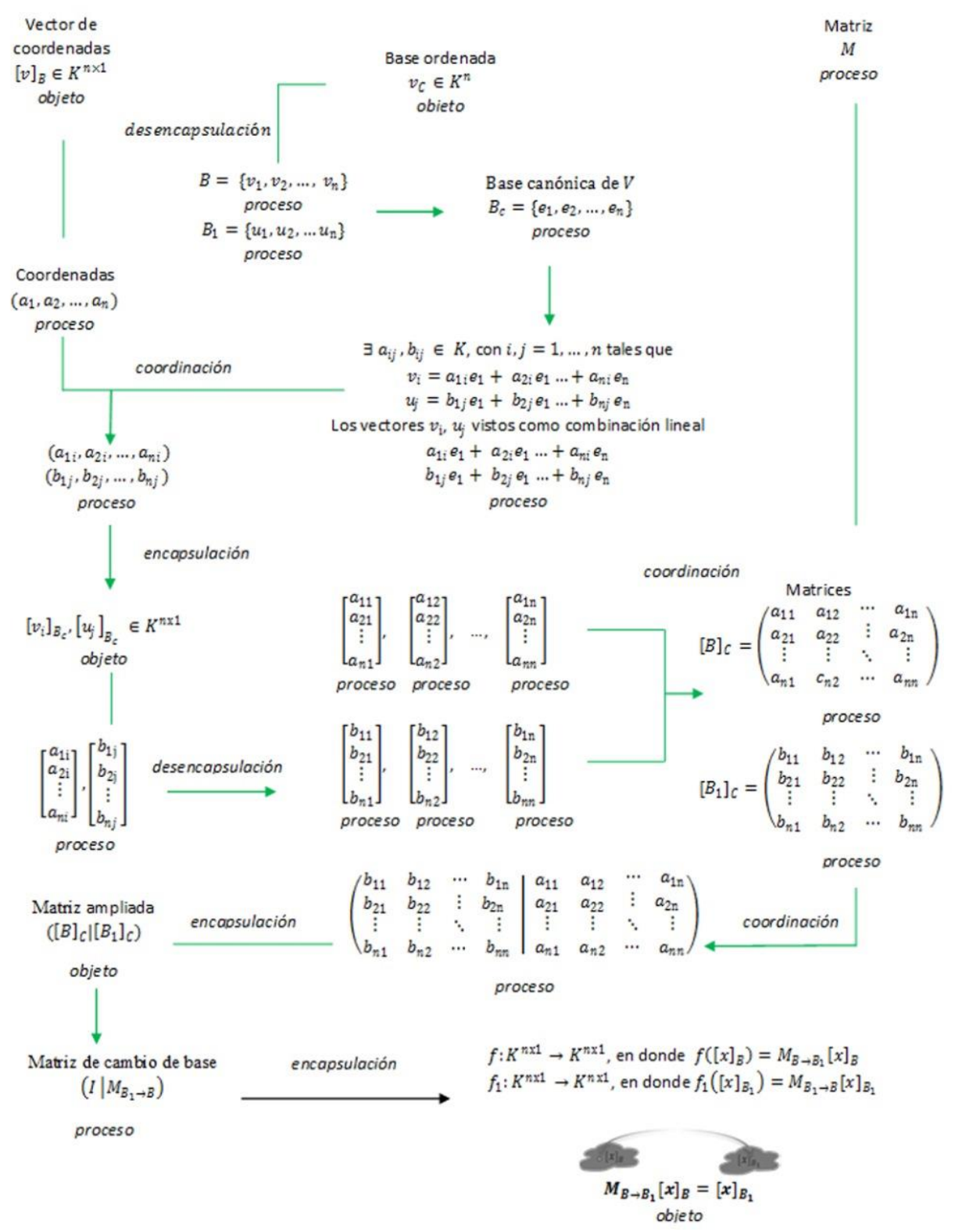

Figura 20. Segunda vía de construcción de matriz de cambio de base

Considerando ambas trayectorias, a continuación, se describe la descomposición genética del concepto de estudio:

La construcción de la matriz de cambio de base (MCB) como un objeto inicia con la desencapsulación del objeto de base ordenada en un proceso, el estudiante con dicha concepción proceso de base ordenada puede escribir los vectores de la base inicial como combinación lineal de los vectores de la base final (según orden de base inicial). Cada proceso de la combinación lineal debe coordinarse con el proceso coordenadas en el proceso coordenadas de un vector y cada nuevo proceso se encapsula en un vector de coordenadas. El objeto vector de coordenadas se desencapsula en el proceso columna de una matriz el cual se coordina con el proceso de matriz en el proceso matriz de cambio de base. En otro caso, el estudiante primero debe identificar el orden en la base para decidir cuál vector va primero, cuál va segundo y así sucesivamente. Luego, se aplican las acciones específicas sobre las bases, escribir las coordenadas de los vectores de ambas bases respecto a la base canónica del espacio vectorial dado. Se coordinan los procesos matriz y vector de coordenadas en el proceso de una matriz ampliada (se colocan 
del lado izquierdo de la matriz los vectores de coordenadas de base final y, del derecho, los vectores de coordenadas de base inicial). Se debe encapsular el proceso en el objeto matriz para aplicar el algoritmo de eliminación de Gauss-Jordan, quedando del lado izquierdo la identidad y del lado derecho una MCB.

Cuando estas acciones se realizan a diferentes bases ordenadas de espacios euclidianos, el estudiante comienza a reflexionar y concibe estas transformaciones como algo dinámico. La interiorización comienza a partir de la reflexión del estudiante sobre los componentes de la MCB, es decir, cuando asimila que cada columna de la matriz está relacionada con la escritura de los vectores de salida como combinación lineal de los vectores de llegada; esto se asocia directamente con las relaciones que establece con las estructuras previas de base ordenada y vector de coordenadas; el estudiante entiende qué representa cada columna de la matriz, se puede predecir el tamaño de la matriz y estar consciente del efecto que causa el orden en las bases. En el caso de considerar las $n$-adas de las bases dadas como columnas, se debe interiorizar que dichos vectores se escriben en una tercera base (usualmente la base canónica del espacio vectorial) además de interiorizar qué matriz va de cada lado para aplicar el método de Gauss-Jordan.

Un estudiante muestra una concepción proceso de la MCB cuando puede calcular correctamente la MCB omitiendo algunas de las acciones específicas anteriores. Además, se tiene control sobre las propiedades y características de la MCB (qué representa cada columna de la matriz; construir una matriz ampliada con los vectores de las bases dadas como columnas; si se cambia el orden en las bases cambia la matriz de cambio de base; la matriz inversa de una MCB es una $\mathrm{MCB}$ ) y se puede calcular correctamente. Asimismo, el estudiante puede distinguir la diferencia entre las matrices cambio de base $M_{B \rightarrow B_{1}}$ y $M_{B_{1} \rightarrow B}$ (diferencia según columnas), y el dinamismo de la estructura proceso de la MCB se presenta en función de las columnas de la matriz y la capacidad de determinar la MCB en cualquier espacio vectorial $V$ de dimensión finita y sobre cualquier par de bases del espacio vectorial $V$. Cuando se tiene necesidad de dejar de pensar en ese dinamismo y se comienza encapsular la MCB entonces se considera a la MCB como una matriz cuadrada y con tamaño relacionado con el espacio vectorial, y además se logra concebir una función definida como el producto de la MCB por una matriz de coordenadas (recibe como entrada un vector de coordenadas en una base y devuelve un vector de coordenadas respecto a otra base) esto es, $f: F^{n \times 1} \rightarrow F^{n \times 1}$ donde $f\left([x]_{B}\right)=M_{B \rightarrow B_{1}}[x]_{B}$ o $f_{1}: F^{n \times 1} \rightarrow F^{n \times 1}$ donde $f_{1}\left([x]_{B_{1}}\right)=M_{B_{1} \rightarrow B}[x]_{B_{1}}$.

Una vez lograda la encapsulación de la MCB, un estudiante con una concepción objeto considera la MCB como un elemento del conjunto de matrices $n \times n$ invertibles, donde $n$ depende de la dimensión del espacio vectorial. Esto se da cuando el estudiante enfrenta situaciones donde necesite aplicar acciones sobre la MCB, por ejemplo, utilizar a la MCB para obtener las coordenadas de un vector respecto a otra base (puesto que ve a la matriz como una regla de correspondencia entre un mismo espacio vectorial).>>

Reconocemos la necesidad de realizar estudios que muestren la encapsulación de la matriz de cambio de base como objeto, pues, aunque dos estudiantes lograron aplicar una acción sobre dicho concepto con éxito, no se alcanzó a concebir el cambio de base como una matriz cuadrada e invertible que describe una función.

\section{Agradecimientos}

Proyectos GUE-2014-01-249670 y C-2018-01 y Programa de Movilidad de la Vicerrectoría de Investigación y Extensión, Universidad Industrial de Santander. 


\section{Referencias}

Anton, H. (1994). Introducción al álgebra lineal. Editorial Limusa. https://doi.org/10.1007/978-1-4614-7966-6

Arnon, I., Cottrill, J., Dubinsky, E., Oktaç, A., Roa, S., Trigueros, M. y Weller, K. (2014). APOS Theory. A framework for research and curriculum development in mathematics education. Springer.

Dubinsky, E. (1991). Reflective abstraction in advanced mathematical thinking. En D. Tall (ed.), Advanced mathematical thinking (pp. 95-123). Kluwer Academic Publishers. https://doi.org/10.1007/0-306-47203-1_7

Florey, F. (1980). Fundamentos de álgebra lineal y aplicaciones. Prentice-Hall.

Grossman, S. (2008). Álgebra lineal. McGraw-Hill.

Hillel, J. (2000). Modes of description and the problem of representation in linear algebra. En J. L. Dorier (ed.), On the teaching of linear algebra (pp. 191-207). Kluwer Academic Publishers. https://doi.org/10.1007/0-306-47224-4_7

Hillel, J. y Sierpinska, A. (1994). On one persistent mistake in linear algebra. En J. P. Ponte y J. F. Matos (eds.), Proceedings PME18 (vol. 3, pp. 65-72). PME.

Hoffman, K. y Kunze, R. (1973). Álgebra lineal. Prentice-Hall.

Montiel, M., Wilhelmi, M. R., Vidadkovic, D. y Elstak, I. (2012). Vectors, change of basis and matrix representation: onto-semiotic approach in the analysis of creating meaning. International Journal of Mathematical Education in Science and Technology, 43(1), 11-32. https://doi.org/10.1080/0020739X.2011.582173

Parraguez, M., Lezama, J. y Jiménez, R. (2016). Estructuras mentales para modelar el aprendizaje del teorema de cambio base de vectores. Enseñanza de las Ciencias, 34(2), 129-150. https://doi.org/10.5565/rev/ensciencias.1950

Poole, D. (2011). Álgebra lineal. Una introducción moderna. Cengage Learning.

Roa-Fuentes, S. y Oktaç, A. (2012). Validación de una descomposición genética de transformación lineal: Un análisis refinado por la aplicación del ciclo de investigación de la teoría APOE. RELIME, 15(2), 199-232.

Selby, C. (2016). Where am I? A change of basis project. PRIMUS. Problems, Resources, and Issues in Mathematics Undergraduate Studies, 26(1), 29-38. https://doi.org/10.1080/10511970.2015.1033796

Trigueros, M. (2005). La noción de esquema en la investigación en matemática educativa a nivel superior. Educación Matemática, 17(1), 5-31.

Trigueros, M., Maturana, I., Parraguez, M. y Rodríguez, M. (2015). Construcciones y mecanismos mentales para el aprendizaje del teorema matriz asociada a una transformación lineal. Educación Matemática, 27(2), 95-124.

Trigueros, M. y Oktaç, A. (2019). Task design in APOS Theory. Avances de Investigación en Educación Matemática, 15, 43-55.

https://doi.org/10.35763/aiem.v0i15.256 
Construcción cognitiva de la matriz de cambio de base

\section{Referencias de los autores}

Esteban Mendoza-Sandoval, Universidad Autónoma de Guerrero (México). emendoza@uagro.mx

Flor Monserrat Rodríguez-Vásquez, Universidad Autónoma de Guerrero (México). flor.rodriguez@uagro.mx

Solange Roa-Fuentes, Universidad Industrial de Santander (Colombia). sroa@matematicas.uis.edu.co

Jesús Romero-Valencia, Universidad Autónoma de Guerrero (México). jesusromero@uagro.mx 


\title{
Study on the cognitive construction of the change of basis matrix in terms of the APOS Theory
}

\author{
Esteban Mendoza-Sandoval, Universidad Autónoma de Guerrero \\ Flor Monserrat Rodríguez-Vásquez, Universidad Autónoma de Guerrero \\ Solange Roa-Fuentes, Universidad Industrial de Santander \\ Jesús Romero-Valencia, Universidad Autónoma de Guerrero
}

In this paper, a construction of the change of basis matrix is proposed, from the viewpoint of APOS Theory, since the abstract nature of Linear Algebra the concepts learning turns out to be weak. Thus, the contribution of this article lies in the analysis of mental structures and mechanisms to obtain a genetic decomposition from conceptions that mathematics students have about the change of basis matrix concept. The cognitive model proposed is the result of the research proposed by APOS Theory, that is, the components of the research cycle are considered: theoretical analysis, data collection and analysis. In this way, in a first time a diagnostic test was given to 28 students (18-21 years old) from Universidad Industrial de Santander, Bucaramanga, Colombia, later, in a second time, 6 from the 28 students were selected for a semi-structured interview. The results show at least two trajectories in the construction of the concept, that is, two ways for its learning, which differ in the interiorization of mental actions. On the one hand, it was found that vectors of the starting basis must be written as a linear combination of the target basis and to interiorize in a coordinate matrix-vector and, on the other, when writing as columns the vectors of the given bases it must be interiorized which should be on the left or the right side of an augmented matrix. These interiorizations intervene and make the difference between the learning ways found and they are considered for the proposed genetic decomposition. Moreover, evidence was found that students which managed to construct the change of basis matrix as a process and that are near to encapsulate the change of basis as an object, show the following mental structures: i) ordered basis as an object; ii) coordinate matrix-vector as an object; iii) matrix as a process. It is worth mentioning that there is no evidence observed about the encapsulation of the study concept, that is, the object structure of the change of basis matrix was not reached, because there are just close ideas when considering the change of basis as an invertible square matrix which describes a function, even though two students successfully managed to apply an action over the change of basis matrix. Finally, in both constructions are manifested the necessary mechanisms and structures for the learning of the change of basis matrix. 